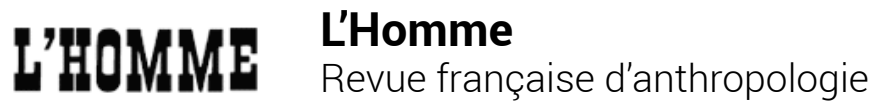

195-196 | 2010

Auto-biographie, Ethno-biographie

\section{Raconter sa vie, raconter des histoires}

\section{Martine Van Woerkens}

\section{OpenEdition \\ Journals}

Édition électronique

URL : http://journals.openedition.org/lhomme/22589

DOl : 10.4000/lhomme.22589

ISSN : 1953-8103

Éditeur

Éditions de l'EHESS

\section{Édition imprimée}

Date de publication : 10 novembre 2010

Pagination : 469-481

ISSN : 0439-4216

Référence électronique

Martine Van Woerkens, «Raconter sa vie, raconter des histoires », L'Homme [En ligne], 195-196 | 2010, mis en ligne le 04 novembre 2012, consulté le 03 mai 2019. URL : http://journals.openedition.org/ Ihomme/22589; DOI : 10.4000/lhomme.22589 


\title{
Raconter sa vie, raconter des histoires
}

\author{
Martine van Woerkens
}

$E_{\mathrm{N}}$ 1998, Stuart Blackburn, historien et tamilophone, responsable des études asiatiques à la School of Oriental and African Studies de Londres, lançait un programme de recherche sur les « histoires de vie». La London School of Economics, les universités d'Oxford et de Cambridge s'associaient au projet. De 1998 à 2000, universitaires britanniques, américains, français, spécialistes d'histoire, de littérature et d'anthropologie de l'Inde se réunirent pour débattre de cette question. Telling Lives in India qui rassemble quelques-uns des meilleurs spécialistes du sous-continent indien est né de ce travail commun. La qualité la plus immédiate de l'ouvrage réside dans l'extraordinaire diversité des vies humaines qu'on y découvre et des formes sous lesquelles ces vies se présentent. Cependant, cette diversité vertigineuse ne serait jamais advenue si cette équipe ne l'avait provoquée, en prenant le contre-pied des cadres conceptuels fondateurs, toujours opérants, du grand partage entre l'Occident et l'Orient.

Cette position critique se déploie sur trois plans. Elle vise tout d'abord un postulat qui a longtemps dominé les études indiennes: l'Inde serait régie par les appartenances de caste, de parenté et de religion et l'explication collective est jugée suffisante pour rendre compte de l'organisation sociale et de ses changements. Pour cette raison, le «je», les notions de pouvoir, d'action et de subjectivité ont été jusqu'à une époque récente occultés. Comment des «dividuals» comme les appelait McKim Marriott (1976, cité p. 2), des gens entièrement déterminés par des logiques collectives, pourraient-ils avoir des histoires de vie individuelles?

À propos de David Arnold \& Stuart Blackburn, eds, Telling Lives in India. Biography, Autobiography and Life History, Delhi, Permanent Black, 2004.

Les traductions des citations sont les miennes. 
Certes, l'anthropologue David G. Mandelbaum écrivait en 1973 un essai pionnier à partir de l'autobiographie de Gandhi, Mes expériences de vérité. Mais il fallut attendre les années 1990 pour que les histoires de vie de personnes moins exceptionnelles (et moins occidentalisées que Gandhi, Nehru, etc.) retiennent l'attention des spécialistes, alors que les études féministes et les black studies exploraient ce genre narratif dès les années 1980. Cette réticence dans les études indiennes témoigne bien du fait qu'on y valorisait la société et non l'individu.

Le premier renversement de perspectives que revendique cet ouvrage consiste à quitter cette vue surplombante du groupe pour rencontrer les individus qui le composent. D'un même mouvement, il s'agit aussi de prendre en compte ceux que l'histoire et l'ethnologie avaient jusqu'alors à peu près ignorés - femmes, basses castes, Intouchables. On aura reconnu dans ce double repositionnement l'influence des études subalternistes ${ }^{1}$, postcoloniales et féministes. Dans la dernière partie de Telling Lives, il est question, justement, d'Intouchables : Viramma, une femme ${ }^{2}$, et Somvaru, un homme. Leurs ethnologues, Josiane et Jean-Luc Racine pour la première, Jonathan Parry pour le second, jouent ici pleinement leur rôle de médiateurs entre deux cultures, entre ces vies jusqu'alors insoupçonnées et nous.

Un autre préjugé, plus général, pèserait aussi dans l'occultation des récits de vie, en Inde comme ailleurs : c'est la conviction que la spécificité culturelle, l'héritage historique de l'Occident seraient la «modernité ». Celle-ci se caractériserait par l'avènement de l'individu, rationnel, capable de réflexivité et agent de ses actions, comme l'attesterait la littérature occidentale depuis Montaigne et surtout Jean-Jacques Rousseau. Là se constateraient l'émergence, le progrès, le développement d'un «je », paré des attributs qui lui conféreraient la dignité biographique ${ }^{3}$. À ce titre, les récits de vie didactiques et illustrant une morale collective qui précède cette "modernité " ne sont pas pris en considération. Or, cette tradition didactique (panindienne et très ancienne, si l'on songe aux multiples vies de Bouddha, des dieux, des saints...) ne s'est pas tarie en Inde et elle côtoie, depuis le XVIII ${ }^{\mathrm{e}}$ siècle jusqu'à aujourd'hui, les formes plus

1. L'historien bengali Ranajit Guha est le fondateur du courant historiographique des subaltern studies. Ce courant marque une rupture avec le paradigme nationaliste de l'historiographie dominante qui, au nom de l'unité nationale, ignorait les luttes de classes. Il constitue ce qu'il est convenu d'appeler The history from below. Depuis 1982, date de publication d'un premier volume collectif, les subaltern studies ont connu un succès considérable en Inde, en Angleterre, aux États-Unis : cf. Pouchepadass (2000).

2. Cf. la monumentale biographie de Viramma, in Viramma, Josiane \& Jean-Luc Racine (1994).

3. Sur cette question de la naissance du biographique au sens «moderne», cf. l'article synthétique de Daniel Fabre (2002). 
occidentalisées de la biographie et de l'autobiographie. Par ailleurs, l'idée que l'on se fait du Soi dans les récits indiens, même récents, les conventions et les modes d'expression utilisés pour l'évoquer, les modèles culturels qui l'inspirent, ne sont pas conformes à notre idée de "modernité ». Est-ce un argument "scientifique " pour tenir à l'écart un si large répertoire?

Telling Lives récuse cette posture: qu'on en juge par l'étude de Barbara D. Metcalf, au chapitre V, sur le récit de vie de Maulana Muhammad Zakariyya (1897-1982)4. Cet héritier d'une grande famille de lettrés musulmans et de saints hommes publia au Pakistan, entre les années 1970 et 1981, sept volumes de plus de deux mille pages. Les traits distinctifs de cette œuvre immense sont bien éloignés de nos critères.

- Tout d'abord, Zachariyya est indifférent à la chronologie, et donc à la notion selon laquelle "la vie fait sens en termes de développements qui créent progressivement une personnalité ou un caractère» (p. 120). La personnalité est avant tout une donnée et, par ailleurs, la meilleure façon de raconter une histoire de vie est de restituer des épisodes qui ont valeur morale.

- Ensuite, le personnage n'identifie pas de buts, ne fait pas de plans, ne surmonte pas d'obstacles; il n'a pas, semble-t-il, de pouvoir d'action ; toute la rhétorique du texte vise à montrer que la forme de sa vie dépend de forces plus grandes que la sienne propre et, par-dessus tout, du pouvoir divin, visible dans le charisme des anciens, morts et vivants, auxquels il doit allégeance.

- Enfin, Zachariyya, loin de se limiter à sa propre histoire, inclut celles de ses aînés, ainsi que l'histoire de ses relations aux autres.

Comparé au modèle occidental "moderne " 5 , Zachariyya se caractérise par la passivité, et son désir de ressemblance avec ses modèles équivaut à se perdre de vue lui-même. Barbara D. Metcalf, l'auteure de l'article qui lui est consacré, défend avec conviction le point de vue inverse. La très forte personnalité du personnage et la passion qu'il déploie pour se soumettre volontairement aux règles exigeantes (celles des aînés), pour voir une signification transcendante dans l'existence quotidienne, pour éprouver enfin la présence d'une communauté morale, représentent au contraire une forme incontestable d'autonomie. Une autonomie dont le moteur n'est pas, comme ici en Occident, quête de la différence.

4. Cf. "The Past in the Present: Instruction, Pleasure and Blessing in Maulana Muhammad Zakariyya's Aap Biitii " (pp. 116-143).

5. Le récit de Zachariyya s'apparente par ailleurs aux modèles biographiques chrétiens prémodernes, didactiques et illustrant une morale collective. 
L'ouvrage de Zacchariyya s'inscrit dans un genre didactique, tazkirah, qui s'appuie sur une tradition dans laquelle l'enseignement de l'islam est incarné par la vie du Prophète et de ses compagnons. Barbara D. Metcalf propose de traduire tazkirah par "mémoire rendue corporelle", car le terme désigne à la fois la mémoire, le moyen mnémotechnique pour se souvenir et la biographie.

En dehors des vies exemplaires et des épisodes à valeur morale, Zacchariyya raconte aussi des épisodes douloureux de son existence. Il intitule son ouvrage Aap-biitii, qui signifie en urdu «ce qui est arrivé à quelqu'un " - une histoire de vie donc, orale et écrite, Zachariyya dictant la plupart des épisodes - mais aussi «témoignage de ses propres souffrances».

Tazkirah et Aap-biitii, qui renvoient tous deux à la biographie mais par des voies si dissemblables, connotent l'intimité de l'islam avec cette production culturelle. Selon que l'histoire de vie se déploie dans le registre du sacré ou du profane, ces deux termes dessinent des champs d'exploration différents, non exclusifs l'un de l'autre, qui s'étirent entre les pôles de la mémoire et de la souffrance, entre la tentative d'incorporation de modèles idéaux et l'expérience banale du malheur.

Si l'on suspendait l'identification du "biographique» avec la "modernité ", Aap-biitii devrait figurer dans le genre que sont les récits de vie, à condition de s'être au préalable débarrassé d'autres conventions encore. C'est le troisième volet de la position critique prise par les auteurs de Telling Lives.

Ces conventions touchent cette fois la littérature, et tout particulièrement ses modes d'analyse et de classement. Ainsi l'analyse de la structure interne du "biographique ", comme on l'a fait avec succès en France et ailleurs à partir d'œuvres occidentales, définit-elle ce genre littéraire selon des critères formels, précis et raffinés, qui, si on les appliquait à Aap-biitii, conduiraient immanquablement à sa seconde exclusion.

Cependant, un nouvel obstacle se dresse pour que l'ensemble des récits de vie soient reconnus comme tels. Il s'agit de la dichotomie entre oralité et écriture, cette autre modalité du grand partage entre l'Occident et les autres. Aujourd'hui, l'histoire orale a acquis droit de cité et l'ethnologie n'est plus confinée aux cultures exotiques et orales. L'opposition est moins entachée par nos idées de primitivisme et de progrès. Cependant, les distinctions disciplinaires dans nos universités font que, selon la nature de leurs supports et selon les usages (historique, ethnologique, littéraire) qu'on en fait, les récits de vie sont éparpillés dans des domaines de savoir séparés. Ils ne pourraient jamais constituer, comme dans cet ouvrage, une entité visible et, partant, un formidable levier pour comprendre les complexités indiennes. 
Telling Lives in India s'affranchit donc de tout carcan formel et conteste le bien-fondé des dichotomies entre oralité et écriture, entre littérature populaire et savante. Le titre insolite de l'ouvrage suggère cette ambition et va même au-delà. Telling Lives, qu'on pourrait traduire par "vies parlantes", est aussi la version abrégée de telling-life histories, qui ellemême rappelle irrésistiblement cette autre expression: to tell stories. Raconter sa vie et raconter des histoires ne seraient donc, si l'on accepte ces associations, qu'une seule et même activité, à l'image du fameux recueil indien, L'Océan des histoires, qui ne raconte que des vies.

L'ouvrage le démontre. Ce flot ininterrompu n'est lié ni à une période historique (les légendes et les contes du Tamilnadu, sans origine précise, étudiés par Stuart Blackburn); ni à un genre (bio- et autobiographie, carnets de prison, journal, etc.) ; ni à un registre (ouvrage édifiant de Zacchariyya, introspection et psychanalyse chez Nehru et M. N. Roy, ou discrétion farouche de l'écrivaine Mahadevi Darma sur sa propre vie, alors que tous ses récits sont écrits à la première personne); ni à la présence d'un, de plusieurs auteurs, ou encore à leur effacement au fil du temps.

Les récits de vie ne sont pas liés non plus à un public déterminé (Somvaru parle à son ami ethnologue Jonathan Parry, Zakira Gouse écrit pour sa famille élargie, Nehru et M. N. Roy pour la nation indienne, Zachariyya pour sa communauté religieuse, etc.); ni à un temps d'élaboration (des années, quelques jours), ni à un mode de création (de la délégation - comme dans le cas de Ananda Ranga Pillai qui commande sa biographie à un tiers - à la provocation - comme dans le cas des enquêtes ethnologiques, etc.); ni au statut de la langue employée (morte ou vivante, savante ou dialectale, etc.); ni à la nature d'un support (écriture, bande magnétique dictée à un tiers qui joue le scribe, parfois supports combinés), ni enfin à un schéma formel (avec un narrateur, un narrataire, un personnage, etc.).

Cette activité humaine aussi multiforme qu'irrépressible représente un océan dans lequel la littérature, telle que les lettrés l'ont définie et asséchée, n'est que l'un des fleuves qui viennent s'y noyer. Car ce qui importe pour les auteurs de cet ouvrage est le noyau de sens, le fait de dire, de raconter des vies.

L'ouvrage récent de Tzvetan Todorov, La Littérature en péril (2007), prône un élargissement des textes retenus au panthéon de la littérature ${ }^{6}$ et déplore que les outils analytiques soient devenus des fins et non plus

6. «Les textes aujourd'hui non littéraires auraient beaucoup à nous apprendre: et pour ma part j'aurais volontiers rendu obligatoire, en classe de français, l'étude de la lettre, hélas guère fictive, que Germaine Tillion adressait depuis la prison de Fresnes au Tribunal militaire allemand, le 3 janvier $1943 »$ (Todorov $2007: 88$ ). 
des moyens pour accéder aux œuvres. Telling Lives anticipe ce double péril et présente tous les bienfaits qu'on retire en l'évitant. Pour comprendre ces formes multiples, tous les "échafaudages" (ibid. : 23 et 86) - histoire, anthropologie, littérature - sont les bienvenus, car le rôle du spécialiste est de faire accéder au sens des récits qu'il présente, de donner à son lecteur la possibilité de "penser et sentir en adoptant le point de vue des autres" (ibid. : 78). Comme Zachariyya qui veut ressembler aux exemples illustres de sa religion, les auteurs de Telling Lives imitent l'art immémorial du conteur et «sa faculté d'échanger des expériences" (Benjamin 2000 : 115).

Que nous apprennent-ils alors sur les récits de vie indiens après les avoir libérés des normes idéologiques, formelles ou disciplinaires? Les présentateurs de l'ouvrage, David Arnold et Stuart Blackburn, situent ces récits à la «frontière de la vérité objective et du récit personnel» (p. 4). Deux composantes de cette frontière retiendront l'attention dans le cadre du présent numéro spécial de L'Homme: la première concerne la véracité du récit, la seconde celle du rapport de l'individu avec la société qu'il met en scène ${ }^{7}$.

\section{La vérité du mensonge}

Comme pour les Bohémiens de Franz Liszt cités dans l'introduction à ce volume ${ }^{8}$, les histoires de vie, écrites ou orales, témoignent de résistances, d'embarras, d'autocensure. Dans son étude, "Honor is Honor, After All" : Silence and Speech in the Life Stories of Women in Kangra " (pp. 227-252), Kirin Narayan relève les ellipses, les abréviations, les codes utilisés par le groupe des femmes âgées, de hautes castes, qu'elle interroge. Il s'agit pour elles de suggérer plutôt que de dire, car la « respectabilité familiale» doit être maintenue. À l'inverse, les femmes plus jeunes, de basses castes, transgressent ces codes, évoquent la violence et les abus sexuels dont elles ont été l'objet à l'intérieur de leur famille. L'honneur de la famille importe moins pour elles "que l'honneur personnel, et donc la vérité ». Les cadres sociaux de la mémoire sont très différents pour ces jeunes femmes qui comptent bien que l'ethnologue sera porteuse, au-delà de leur village, des atrocités qu'elles ont subies, et pour ces femmes plus âgées prises dans une tension souvent repérable, entre «le désir intense de dire la vérité et le désir tout aussi intense de la contrôler» (p. 17).

7. Sur ces deux questions, cf. Artières \& Kalifa (2002).

8. Voir supra, p. 9. 
Dans «The Marital History of "A Thumb-Impression Man” " (pp. 281318), Jonathan Parry apporte un autre témoignage de cette tension. Somvaru, son informateur et ami, est doué d'une mémoire aussi précise qu'extraordinaire. Par ailleurs, il est sincère: "Mon corps ne peut pas mentir" (p. 87). Il n’empêche que le récit de sa "vie maritale", certes exubérante, n’est ni linéaire ni immédiate : si la première année, l'ethnologue apprend l'un après l'autre les quatre mariages de Somvaru, il ne prendra connaissance que trois ans plus tard du cinquième. Enfin, ce n'est qu'au bout de huit ans que Somvaru lui révèle que sa « fille " Janaki n'est pas sa fille biologique.

Jonathan Parry évoque cette autre situation où l'informateur sincère raconte quelque chose que l'ethnologue tient pour une fiction, ainsi lorsque Somvaru parle de son enfance et de sa rencontre avec une horde terrifiante de sorcières alors qu'il conduisait une charrue. Pour Parry, la contradiction est plus apparente que réelle. Car cette rencontre fantastique avec des sorcières est la chose la mieux partagée par les habitants de la région de l'enquête. Ces récits puissants, appris dans l'enfance, sont intériorisés comme s'ils avaient été vécus. Ils sont stockés comme des modèles mentaux qui permettent d'imaginer qu'on y était. Il y a une "vérité du mensonge " et c'est elle que l'ethnologue interroge aussi.

Par ailleurs, Parry observe que le travail de mémoire n'est pas homogène. Il y a ces histoires qui semblent proches de la réalité et que Somvaru relate plusieurs fois exactement de la même manière. Ces souvenirs semblent être "en pilotage automatique». On ne s'en souvient pas, on se souvient seulement de la manière dont on les a racontés. Et puis, il y a les matériaux bruts, incomplètement fixés en souvenirs et souvent provoqués par les questions. On s'en souvient plus qu'on ne se les rappelle.

Telle qu'elle apparaît dans les carnets de l'ethnologue, l'histoire de vie de Somvaru est un patchwork de récits qui se déploient au cours de huit années: "Certains sont représentés de manière formelle et conventionnelle, d'autres sont des versions intériorisées d'histoires tirées d'un vaste répertoire culturel, d'autres encore sont des rappels de rappels antérieurs, et d'autres enfin, des souvenirs jusqu'alors jamais verbalisés " (pp. 285-286).

Non seulement le récit de vie est situé - c'est un point de vue proféré dans des circonstances particulières et qui s'adresse à une personne particulière -, et il serait naïf de l'ignorer, de surcroît, selon l'objet dont se saisit la mémoire, il puise dans des sources différentes, revêt des formes diverses, contredisant éventuellement la sincérité du narrateur. 
Les auteurs des Veda, nous apprend Charles Malamoud (2005), recommandent de ne pas mentir. Mais ils savent aussi que vérité et mensonge sont intimement liés, car la parole de l'homme est "adéquate au monde et au non-monde, à ce qui est et à ce qu'il veut» (p. 85). Enfin, toujours selon ces textes, "le mensonge est la racine de la parole» (p. 87), au sens où il expose la vérité la plus intime du menteur. Les ethnologues de Telling Lives partagent cette sagesse: la vérité racontée a de multiples facettes. Le faux et le vrai ne se valent pas, mais l'un et l'autre sont porteurs d'enseignements. Le "noyau de sens" n'est pas une épure (et donc un mensonge !) dans laquelle l'homme aurait perdu «cette aptitude à ne pas être totalement dans le présent " et ne serait plus "doué de mémoire et d'imagination anticipante" (ibid. : 85 ).

"Qu'est-ce que peut être une science écrite des cultures non écrites?", interroge Jack Goody dans La Raison graphique? ${ }^{9}$ Jonathan Parry prend le parti d'observer la palette des mémoires mises en œuvre par Somvaru, de telle sorte que les plans de la réalité semblent disjoints. Ils se situent autant dans l'imaginaire que dans le monde réel, dans l'improvisation que la répétition, dans l'approximation que la précision. C'est par ces voies multiples que le « récit personnel» se fraye un chemin.

\section{Individu et société}

Dans leur introduction, qui est aussi le bilan des travaux de l'équipe sur les «histoires de vie ", David Arnold et Stuart Blackburn affirment qu'en Inde, aucune de ces histoires ne parle de vie purement isolée, autonome, individuelle. Il arrive que le personnage agisse de lui-même, reconnaissentils, mais toujours en rapport avec d'autres qui se définissent, eux aussi, par leur relation à des cadres de référence plus larges (famille, caste, religion, genre) et par leur relation à ces forces encore plus puissantes que sont le nationalisme, la modernité, le colonialisme, l'industrialisation, etc.

"L'image du réseau, de l'individu lié à de nombreux autres, par des alliances de plus ou moins grande intensité et intimité »(p. 22) visualise au mieux l'hypothèse présentée dans ce volume. Au questionnement traditionnel (comment la société prend en charge les vies individuelles ?) s'est substitué son symétrique inverse. Mais ce renversement n'aboutit pas à la découverte, là-bas, de notre "modernité». En effet, ce que ces récits de vie mettent en scène, c'est de manière "nuancée et subtile ", The Selfin-Society, et cette société n'est pas la nôtre.

9. La citation vient de l'introduction (Goody 1979: 14). Telling Lives in India, à bien des titres, prolonge la réflexion de cet ouvrage, très novateur à l'époque, sur les processus de connaissance mis en œuvre dans l'oralité et l'écriture et qui replace le travail ethnographique dans le passage critique de l'oral à l'écrit, ce "corpus achevé et immuable " après le gommage "des processus de création et de reformulations permanentes" (Ibid.). 
Les intersections de la vie de Somvaru avec ces cercles d'appartenance de plus en plus larges, au fur et à mesure qu'on s'éloigne de la sphère privée pour entrer dans le flot de l'Histoire, illustrent cette «image du réseau ". On y retrouve bien sûr la caste. Mahar et paysan, traditionnellement affecté à l'équarrissage du bétail et au travail du cuir, ce citadin de la township industrielle de Bhilai vit dans le quartier "intouchable" du village et se soumet aux rites d'expiation coutumiers (offrir un repas aux membres de sa caste) lorsque sa conduite sexuelle outrepasse les règles, pourtant fort lâches, toutes castes confondues, dans cette région indienne.

La parenté, aussi menacée soit-elle dans ce nouveau contexte urbain, joue un rôle fondamental dans quatre de ses cinq mariages : le premier, qui a lieu alors qu'il est enfant et dont il ne se souvient plus, est arrangé par des aînés; les autres s'accomplissent, à sa demande, grâce à l'aide des alliés et parents de sa génération. Sa relative aisance économique, enfin, provient en partie de la politique industrielle menée par Nehru après l'indépendance de l'Inde.

Mais ces déterminismes ne brident pas sa capacité d'autonomie et son désir d'inventer sa vie. En témoigne son positionnement très personnel et très affirmé au sein de sa société. Malgré les drames qui jalonnent son existence - les morts nombreuses, à commencer par celle de ses parents quand il était enfant -, malgré les stigmates attachés à son statut, malgré son illettrisme, malgré tous ces handicaps, Somvaru n'est pas simplement le jouet des circonstances. Il en est aussi parfois le bénéficiaire.

Contre la pauvreté, il improvise en se lançant dans de nombreuses entreprises aux quatre coins de l'Inde: avec ses amis, il part à Calcutta, achète des ustensiles de cuivre et les revend; puis, il fait le transport de bambou et de teck à partir des jungles de Dongargarh; puis, il découvre qu'acheter du bétail au Maharashtra et le revendre dans sa région représente un meilleur profit. Il occupe aussi différents postes au sein de Bhilai Steel Plant, et survit, dit-il, en perruquant ${ }^{10}$, c'est-à-dire en adoptant des tactiques de détournement (absentéisme, vols, etc.) contre les intérêts de la compagnie. Son enrichissement ne repose d'ailleurs pas exclusivement sur son travail, il joue à l'occasion les prêteurs d'argent et tire profit de l'heureuse convergence entre ses racines paysannes, qui le poussent à posséder de la terre pour la cultiver comme ses ancêtres avant lui, et l'expansion économique extraordinaire de la township de Bhilai. Un jour, les terres qu'il cultivait deviennent des terrains à bâtir. Somvaru se retrouve alors à la tête d'un capital non négligeable. Il n'est plus pauvre parmi les pauvres. 
Humilié d'être un illettré, l'« homme qui signe avec le pouce » (Thumb Impression Man) comme l'appelle cruellement l'un de ses fils fait en sorte que tous ses enfants, sauf un, sachent lire et écrire. Sa fille Janaki enseigne même au lycée. Cependant, l'«illettré» dispose de multiples savoirs; il est cultivé. Il est l'une des vedettes d'une troupe de musiciens et de chanteurs professionnels; il compose et chante des chansons, et part régulièrement en tournée dans les villages alentour. Par contraste avec Viramma ${ }^{11}$ ("Nous sommes des parias... il faut être humble, rester à sa place» p. 287), il méprise tranquillement «les grands airs de ceux pour qui la supériorité va de soi » (p. 287) et exerce son sens critique dans ses chansons. Il n'embrasse à aucun moment la position de victime, ni à l'égard de la société ni à l'égard de ses enfants, pourtant guère tendres avec lui. Il ne cède pas non plus aux pressions de son beau-fils Barle, le mari de Janaki, qui veut le forcer à vendre des terrains. Avant de disparaître pendant plusieurs semaines, Somvaru lui répond: "C'est vrai. Je ne suis pas éduqué. Mon père ne m'a pas éduqué. Mais c'est ma terre. Je la vendrai à qui je veux» (p. 293).

«Somvaru n'habite pas le monde crépusculaire de Muli ${ }^{12}$, et il differe aussi de Vasant Moon, un intellectuel dalit d'un segment très politisé d'une caste très politisée, auteur d'une récente biographie ${ }^{13}$ " (pp. 286287). Sans doute né en $1935^{14}$, âgé de quelque soixante-dix ans au moment de l'enquête, pilier des nombreuses familles recomposées qu'il a formées, Somvaru, ni résigné ni révolutionnaire, contemple avec "détachement» les bouleversements de son existence et les changements vertigineux qui affectent celle de certains de ses enfants.

Pourquoi ce détachement? Est-ce à cause de la tradition culturelle hindoue qui demande qu'à cette étape de la vie on abandonne " tout désir de désir " ? Plusieurs hypothèses sont écartées les unes après les autres. La réponse serait-elle dans le fait que Somvaru apprécie par-dessus tout les hommes capables d'avoir la "tête froide", thanda dimag, par rapport à leur sexualité, leurs émotions, leur vie maritale? Parry ne conclut pas. Il souligne combien cet idéal vers lequel tend Somvaru "connote fortement l'idée d'une retenue civilisée et d'une amabilité paisible» (p. 311).

11. Viramma, évoquée plus haut, est le nom de la femme intouchable dont Josiane et Jean-Luc Racine ont établi la biographie.

12. Muli est le nom de l'Intouchable dont James M. Freeman a établi la «crépusculaire» biographie (1979).

13. Cf. Moon (2001).

14. Les pauvres ne déclarent pas la naissance de leurs enfants en Inde. L'état civil de manière générale est assez lâche : très souvent, c'est l'inscription à l'école ou le besoin d'un passeport qui détermine cette démarche administrative. 
Dans cet article remarquable où l'ethnologue réfléchit tout haut et où les «mensonges" de l'informateur sont tissés dans l'enquête, Jonathan Parry propose un ultime rebondissement. Il mérite d'être signalé au terme de ces pages dans lesquelles je défends le point de vue des auteurs de Telling Lives. Car Parry apporte un argument supplémentaire, et essentiel, à son ethnologie fondée sur le récit de vie. En effet, s'il souscrit au pari général de l'ouvrage - l'intérêt est passé « de la structure au pouvoir d'agir, de la culture à la voix individuelle, du traditionnel au monde globalisé " (p. 283) -, c'est parce que, dit-il, le terrain l'y contraint. C'est pour être au plus près de la réalité sociale nouvelle à laquelle il est confronté à la fin des années 1990 en Inde :

«En réfléchissant à mes terrains antérieurs, dans un village kangra [...] et parmi les "spécialistes sacrés" de la ville de Bénarès [...], je suis frappé par le contraste. Là-bas mes informateurs étaient très volontiers prêts à me dire les règles et les traditions. Et la plupart étaient embarrassés lorsque mes questions portaient directement sur leurs pratiques, sur qui avait fait quoi. Je m’attendais à la même chose en commençant ce terrain. Mais ce n'est pas ce que j'ai trouvé. Ici [dans la région de Chhattisgarh où vit Somvaru], les gens ne prêtent aucun intérêt aux règles, cela les rend impatients. Et même ceux que je venais juste de rencontrer me donnaient très volontiers des informations très précises sur leur vie ou même sur celle de leurs voisins. Ce dont ils voulaient vraiment parler étaient les événements, pas les structures.

Pourquoi ? Il faudrait écrire un article pour répondre. Mais voici un début de réponse : Une "grande transformation" a radicalement bouleversé le monde dans lequel étaient nés mes informateurs les plus âgés, les mélangeant à des personnes socialement et régionalement différentes. Le vieil ordre a été relativisé, il s'est avéré éphémère, et personne ne le regrette. Beaucoup de ces individus sont au plus bas de l'échelle sociale, et leur investissement dans ce vieil ordre n'est sans doute pas très élevé. Ils vivent de surcroît dans un monde consciemment en train de se moderniser, au sein d'un de ces géants industriels du secteur public de l'ère nehruvienne, spécifiquement pensé pour anéantir le passé. Beaucoup ont intériorisé ces nouvelles valeurs. Mais le plus important est l'échelle vertigineuse des changements dont ils ont été les témoins. Face à ces changements, subordonner les événements à des structures intemporelles, faire coïncider des vies individuelles avec des trajectoires biographiques standards et dépersonnalisées, serait de la naïveté [...]. Ces événements rendent impossible de croire que, tel "un roc au milieu du fleuve" ${ }^{15}$, une personne se définirait par une quelconque essence sur laquelle le temps et les circonstances ne laisseraient aucunes traces » (pp. 283-284).

Somvaru, tel que le raconte Parry, apparaît comme un Intouchable postmoderne. Il ne pourrait ni figurer dans les répertoires de "types et caractères" chers au XIX siècle, ni être l'informateur privilégié d'un

15. La citation est de Maurice Bloch ( Internal and External Memory: Different Ways of Being in History", in How We Think They Think. Anthropological Approaches to Cognition, Memory, and Literacy, Oxford, Westview Press, 1998 : 67-84). 
ethnologue du XXe siècle qui souhaiterait écrire une monographie sur les "Intouchables du Chhattisgarh». Il est unique. Comme l'écrit son biographe et ami, «les vies des Intouchables ne sont pas plus uniformes que celles des autres couches de la société» (p. 287).

Cela signifie que notre humanité est faite de singularités aussi remarquables les unes que les autres. C'est ce que révèlent toutes les histoires de vie étudiées dans ce volume et, entre autres, celles de Zachariyya et de Somvaru évoquées ici plus en détail. Telling Lives serait donc un exercice pratique, une mobilisation accomplie de l'idéal des Lumières, l'unité du genre humain.

Mais l'Occident a récemment contesté cette unité.

Par principe de précaution, je suggère donc d'adopter un point de vue non occidental sur cette question, celui que propose Ashis Nandy dans L'Ennemi intime. Perte de soi et retour à soi sous le colonialisme (2007). Il y parle d'"universalisme alternatif». Alternatif, parce qu'il s'agit, explique-t-il dans son ouvrage, de la version locale, indienne, de cette unité. Ce sont, je crois, les limites positives de l'horizon de Telling Lives in India.

École pratique des hautes études Section des sciences religieuses, Paris vanwoerkens@wanadoo.fr

MOTS CLÉS/KEYWORDS : biographie/biography - Inde/India - études subalternistes/subaltern studies - Intouchables/Untouchables - postmodernisme/postmodernism. 
Artières, Philippe \& Dominique Kalifa, eds

2002 Sociétés et Représentations 13 :

Histoire et archives de soi. Paris, CREDHESS.

Benjamin, Walter

2000 «Le conteur: réflexions sur l'œuvre de Nicolas Leskov [1972]", in CEuvres, 3. Paris, Gallimard («Folio»).

Certeau, Michel de

1990 [1980] L'Invention du quotidien, 1 : Arts de faire. Paris, Gallimard ("Folio»).

Fabre, Daniel

2002 "Vivre, écrire, archiver", Sociétés

et Représentations 13: Histoire et archives

de soi: 19-42.

Freeman, James $\mathrm{M}$.

1979 Untouchable. An Indian Life History. Stanford, Stanford University Press.

\section{Goody, Jack}

1979 La Raison graphique.

La domestication de la pensée sauvage.

Trad. et prés. de Jean Bazin et Alban Bensa.

Paris, Minuit ( Le sens commun»).

\section{Malamoud, Charles}

2005 «L'arbre aux racines de mensonge: variations indiennes sur le vrai et le faux ", in Féminité de la parole. Études sur l'Inde ancienne. Paris, Albin Michel ("Sciences des religions) : 73-91.

\section{Mandelbaum, David G.}

1973 "The Study of Life History:

Gandhi ", Current Anthropology 14 (3) : 177-196.
Marriott, McKim

1976 «Hindu Transactions: Diversity without Dualism ", in Bruce Kapferer, ed., Transaction and Meaning. Directions in the Anthropology of Exchange and Symbolic Behavior. Philadelphia, Institute for the Study of Human Issues : 109-142.

Moon, Vasant

2001 Growing up Untouchable in India. A Dalit Autobiography. Transl. from the marathi by Gail Omvedt.

Lanham, Rowman \& Littlefield.

Nandy, Ashis

2007 L'Ennemi intime. Perte de soi et retour à soi sous le colonialisme. Trad. de l'anglais (Inde) par Annie Montaut. Préf. de Charles Malamoud. Paris, Fayard («Les quarante piliers »). [Éd. orig. : The Intimate Enemy. Loss and Recovery of Self under Colonialism, Delhi, Oxford University Press, 1983.]

Pouchepadass, Jacques

2000 "Les subaltern studies, ou la critique postcoloniale de la modernité ", L'Homme $156: 161-186$.

\section{Todorov, Tzvetan}

2007 La Littérature en péril.

Paris, Flammarion ("Café Voltaire»).

Viramma, Josiane \& Jean-Luc Racine

1994 Une vie paria. Le rire des asservis: pays tamoul, Inde du Sud. Paris, Plon-Unesco («Terre Humaine »). 